\title{
Preschoolers sometimes know less than we think: The use of quantifiers to solve addition and subtraction tasks
}

\author{
BELINDA BLEVINS-KNABE \\ University of Arkansas at Little Rock, Little Rock, Arkansas \\ ROBERT G. COOPER \\ Southwest Texas State University, San Marcos, Texas \\ PRENTICE STARKEY \\ University of California, Santa Barbara, California \\ PATTY GOTH MACE \\ Child and Family Services, Hartford, Connecticut \\ and \\ ED LEITNER \\ Puyallup, Washington
}

(John B. Pittenger, Sponsor)

\begin{abstract}
This study addressed the issue of whether young children's success on small numerosity tasks is due to the use of perceptually based quantifier skills, such as subitizing, or to their precocious understanding of small numbers. Children 5 years of age were given a series of addition and subtraction trials that included large and small numerosity trials as well as trials on which the children were asked to imagine that objects were present. The imaginary trials had the same number of items as the small numerosity trials. Because perceptual representations were not available on the imaginary trials, the children's solutions could not result from the use of perceptual quantifier skills. Performance on all imaginary trials was similar to performance on the large numerosity trials, and poorer than that on the small numerosity trials. Because it was difficult for young children to quantify on either large numerosity trials or imaginary trials, we conclude that our subjects' superior performance on small numerosity trials was due to their use of quantifier skills, rather than to a precocious understanding.
\end{abstract}

Many investigations of number concepts have found that young children's performance is affected by numerosity size. When young children are given both small (one to four items) and large (over four items) numerosity tasks, they perform better on the former (Apostel, Mays, Morf, \& Piaget, 1957, cited in Flavell, 1970; Bryant, 1974; Gelman, 1972; Winer, 1974). Several current models of the development of numerical understanding predict these findings. Although there have been different interpretations of Piaget's (1952) theory, one is that children apply numerical operations first to small numerosities and later to large ones (Flavell, 1970). Gelman (1982) demonstrated that although young children can, in limited contexts, deal with numerosities of unspecified values, they are more

Preparation of this article was supported by the Marie Wilson Howells' bequest to the Psychology Department, University of Arkansas at Little Rock. We would like to thank Linnea Michaels for assistance in the collection of the data. Requests for reprints should be sent to the first author at the Department of Psychology, University of Arkansas at Little Rock, 33rd and University, Little Rock, AR 72204. likely to be successful in a wider variety of contexts on tasks with small numerosities. According to Gelman's model, children have an implicit understanding of some number concepts, and what develops is a more general ability to access this implicit understanding (Gelman, 1982; Gelman \& Gallistel, 1978). Young children's superior performance on small numerosity tasks is taken as support for the accessing model.

Siegler's (1986; Siegler \& Robinson, 1982) model of numerical understanding also addresses the issue of the understanding of small versus large numerosities. According to this model, children develop the correct rule for solving a problem such as small number conservation before they develop it for a problem such as large number conservation. The more advanced rules, such as those for large number conservation, develop from the less advanced rules.

In Gelman's model, the assumption is that correct performance on small numerosity tasks is an indication of the children's understanding of numerical concepts, rather 
than an indication of their use of quantifier skills such as counting or subitizing. Cooper (1984) referred to skills used to establish numerosity as quantifiers and to numerical concepts such as conservation, addition, and subtraction as operators, and we will use that terminology here. In Siegler's model it is not possible to tell whether children's solutions to small numerosity problems are based on quantifier solutions or operator solutions (Siegler \& Robinson, 1982). It is necessary to specify the basis of the solution of small numerosity problems if we are to understand the transition from correct performance on small numerosity tasks to correct performance on large numerosity tasks. Information about this transition would serve to either support or refute Gelman's accessing theory and to explicate Siegler's and Piaget's (1952) theories.

An understanding of this transition is also critical to a third model of numerical understanding, which is focused on addition and subtraction. In a series of studies on the development of addition and subtraction understanding in preschool children (Blevins, Mace, Cooper, Starkey, \& Leitner, 1981; Cooper, 1984; Cooper, Campbell, \& Blevins, 1983; Cooper, Starkey, Blevins, Goth, \& Leitner, 1978), we have found support for three levels of addition and subtraction understanding. In each study, however, when we asked children to make relative numerosity judgments, we found that they succeeded on small numerosity trials before they succeeded on large numerosity trials.

We call the three levels of understanding the primitive, qualitative, and quantitative levels. At the first, or primitive, level, children attend only to the transformation when making a decision about relative numerosity. For example, if a subtraction is made to one of two arrays, the children will say the array has less, or if an addition is made, they will say the array has more. Because in our studies additions and subtractions occurred only to equal arrays or to arrays that differed by no more than two items, these responses were correct only when the initial two arrays were equal. At the second, or qualitative, level, children attend to both initial state and transformations. One array is coded as being less than, equal to, or greater than the other. An addition changes a "less than" relation to an "equal to" relation, and a subtraction changes a "greater than" relation to an "equal to" relation. If the initial arrays are equal, then an addition to an array causes it to have more, and a subtraction causes it to have less. At the third, or quantitative, level, children quantify both the initial differences between arrays and the transformation, and thereby arrive at fully accurate performance (see Table 1).

We found that children's solutions on the large numerosity trials comprised a developmental progression from the primitive level to the qualitative and quantitative levels, but their solutions on the small numerosity trials were correct regardless of trial type. If accurate performance on the small numerosity trials is an indication of children's implicit understanding of addition and subtraction, then models like those of Gelman or Siegler can explain the developmental changes that take place. However, children may be successful on small numerosity tasks not because they are making use of implicit understanding of the principles involved, but because they are using quantifiers such as counting or subitizing. Since there is evidence that 3-year-old children can subitize numerosities up to four (Chi \& Klar, 1975; Cooper \& Starkey, 1977), we hypothesized that correct performance on our addition and subtraction tasks might be due to the use of this quantifier and not to the use of operators.

We tested this hypothesis by using trials from a previous addition and subtraction study conducted with 3-, 4-, and 5-year-olds (Cooper et al., 1978). In that study, transformations occurred to two arrays and the children were asked to make a relative numerosity judgment. These types of trials proved to be more difficult for the children than those used in our other studies, in which we performed one transformation, asked for a relative numerosity judgment, and then performed a second transformation and asked for a second judgment. Because the type of procedure we chose for the present study was so difficult, we tested only 5-year-olds and included only primitive and qualitative trials. A new condition, called the imaginary condition, was included to prevent the use of a perceptually based quantifier skill such as subitizing. In this condition the children had to imagine that objects were present. Because no objects were actually present, the children could not subitize. The children were asked not to count and the experimenter interrupted any overt counting that took place.

Table 1

Predicted Solutions to Trials by Addition and Subtraction Reasoning Levels

\begin{tabular}{ccccccc}
\hline & Trial Structure & & & \multicolumn{3}{c}{ Reasoning Levels } \\
\cline { 1 - 2 } Initial State & Transformation & Final State & & Primitive & Qualitative & Quantitative \\
\hline$N$ & +1 & $N+1$ & & Correct & Correct & Correct \\
$N$ & +0 & $N$ & & & & \\
$N$ & +1 & $N+1$ & $N+1>N+1+0$ & Correct & Correct \\
$N+1$ & +0 & $N+1$ & & & \\
$N$ & +1 & $N+1$ & & $N+1>N+2+0$ & $N+1=N+2$ & Correct \\
$N+2$ & +0 & $N+2$ & & & & \\
\hline
\end{tabular}

Note $-N$ means that arrays are initially equal. 


\section{METHOD}

\section{Subjects}

Sixteen five-year-olds with a mean age of 65 months participated. The 5 girls and 11 boys came from middle socioeconomic backgrounds in Austin, Texas.

\section{Design}

Each subject received an addition and subtraction task followed by a conservation task. On the addition and subtraction task the withinsubjects variables were type of trial (primitive or qualitative) and type of set (small, 2 to 4 items; large, 6 to 10 items; imaginary, 2 to $4 \mathrm{im}$ aginary items). The within-subjects variables on the conservation task were numerosity (small and large) and type of transformation (standard and nonstandard).

\section{Procedure}

The subjects were tested individually, each in one 30-min session, which included a warm-up task, the addition and subtraction task, and the conservation task. There were $\mathbf{1 8}$ addition and subtraction trials, 3 of each of the following types: imaginary primitive, imaginary qualitative, small primitive, small qualitative, large primitive, and large qualitative (see Table 2). The trials were arranged in random order, with the provision that a small numerosity task come first to facilitate performance. The children were asked not to count on any task and the experimenter interrupted any overt counting that occurred. The warm-up task demonstrated that all of the children understood the terms same and most.

The procedure for all addition and subtraction trials was the same. The children were shown two small round petri dishes and were told that the experimenter would be placing pinto beans in them. The experimenter then placed the beans in the dishes in a manner emphasizing one-to-one correspondence. To start, one bean was placed simultaneously in each dish. This was repeated until the initial arrays had been constructed. As the beans were placed into the dishes, the experimenter said, "I'm putting one here and one here." If the initial arrays were unequal, the experimenter continued to place beans one at a time in one dish until it contained the correct number. The beans were arranged randomly in the dishes. The experimenter then asked the following standard question: "Does this one have the most, or does this one have the most, or do they have the same number of beans?" The superlative most was used because pilot work demonstrated that children understood it better than the term more. If the child answered this question incorrectly, the experimenter removed the beans and started over. If the child answered correctly, the experimenter performed successive transformations on the arrays. For example, in the unequal subtraction transformation the experimenter removed two beans from one dish, one at a time, and said, "See, I'm taking one from this dish. Now I'm taking

Table 2

Trial Structures Used in the Addition and Subtraction Task

\begin{tabular}{|c|c|c|}
\hline Initial State & Transformation & Final State \\
\hline \multicolumn{3}{|c|}{ Primitive Trials } \\
\hline $\begin{array}{l}N \\
N+1\end{array}$ & $\begin{array}{l}+1 \\
+2\end{array}$ & $\begin{array}{l}N+1 \\
N+3\end{array}$ \\
\hline $\begin{array}{l}N+1 \\
N\end{array}$ & $\begin{array}{l}-1 \\
-2\end{array}$ & $\begin{array}{l}N \\
N-2\end{array}$ \\
\hline $\begin{array}{l}N \\
N\end{array}$ & $\begin{array}{l}+1 \\
-1\end{array}$ & $\begin{array}{l}N+1 \\
N-1\end{array}$ \\
\hline \multicolumn{3}{|c|}{ Qualitative Trials } \\
\hline $\begin{array}{l}N \\
N+1\end{array}$ & $\begin{array}{l}-1 \\
-2\end{array}$ & $\begin{array}{l}N-1 \\
N-1\end{array}$ \\
\hline $\begin{array}{l}N \\
N+1\end{array}$ & $\begin{array}{l}-1 \\
-2\end{array}$ & $\begin{array}{l}N-1 \\
N-1\end{array}$ \\
\hline $\begin{array}{l}N \\
N+2\end{array}$ & $\begin{array}{l}+1 \\
-1\end{array}$ & $\begin{array}{l}N+1 \\
N+1\end{array}$ \\
\hline
\end{tabular}

another one," and then removed one bean from the other dish, saying, "See, I'm taking a bean from this dish." The child was asked the standard question and the trial was completed.

The procedure on the imaginary trials was the same except that the children were told that the experimenter had "pretend beans" that they could not see. The experimenter performed the same gestures as when real beans were used, and the arrays were established and transformations were performed with temporal one-to-one correspondence cues available.

The conservation task included small (two items) and large (eight items) numerosity trials and a standard and nonstandard transformation for each numerosity. The small numerosity trials were given first. The standard transformation was an expansion or contraction of one of the arrays. The nonstandard transformation involved moving one internal item to the end of the array so that the array had both a length cue and a gap cue. In distinction to the standard transformation, which highlights misleading length cues, the nonstandard transformation places the length cue in conflict with the gap cue (cf. Bryant, 1974). In the small numerosity trial, one of the items was pushed over to the side. The children were asked for explanations on all trials.

\section{RESULTS}

An examination of performance on the addition and subtraction trials revealed that the children performed better on the primitive trials than on the qualitative trials, except on the small numerosity trials. An analysis of variance with the between-subjects factors of type of set (small, large, imaginary) and type of trial (primitive and qualitative) was performed. There were main effects for numerosity $[F(2,30)=29.14, p<.001]$ and trial type $[F(1,15)=87, p<.001]$, as well as a numerosity $\times$ trial type interaction $[F(2,30)=21.56, p<.001]$. Newman-Keuls tests $(p<.05)$ revealed that, with one exception, all of the qualitative trials were more difficult than the primitive trials. The small numerosity qualitative trials were not different from the primitive trials. The children's good performance on all of the primitive trials, including the imaginary trials, indicates that they were able to understand the experimenter's instructions about the imaginary task.

The conservation data were analyzed by examining both judgments and explanations. Performance on the conservation tasks was high. The children were $97 \%$ correct on the small numerosity judgments and $82 \%$ correct on the large numerosity judgments. However, there were no significant differences between small and large numerosity trials or between standard and nonstandard transformations. Seven children gave at least one type of adequate explanation for their correct judgments. Adequate explanations included those based on reversibility, lack of addition or subtraction, compensation, or irrelevancy of the transformation.

Because most of the children did well on the conservation task, it is not possible to make meaningful comparisons between level of performance on the conservation task and level of performance on the addition and subtraction trials. However, the data from the present study are consistent with those from the previous study in which we employed the same type of trials (Cooper et al., 1978); both studies showed that good conservation performance 
did not ensure good performance on the qualitative trials. The trials used in these studies were more complex than the addition and subtraction trials used in the majority of our studies, which involved sequential transformations and in which we found a qualitative understanding to be necessary, but not sufficient, for conservation.

\section{DISCUSSION}

The results of this study indicate that children can use operator solutions on large and imaginary trials when the basis of a primitive understanding. To be successful on these primitive trials, the children had only to attend to the transformation. However, the children's success on the small qualitative trials and failure on the large qualitative and imaginary qualitative trials suggests that on these trials they relied on quantifier solutions rather than operator solutions, because an operator solution would involve the use of a qualitative rule that integrates an initial state and a transformation. Subitizing is the quantifier most likely to have been used on these trials, because overt counting was prohibited and because subitizing would produce correct answers on small numerosity tasks, but not large or imaginary tasks.

These results have a clear implication for models of number development such as those of Gelman (Gelman, 1982; Gelman \& Baillargeon, 1983; Gelman \& Gallistel, 1978) and Siegler (Siegler, 1986; Siegler \& Robinson, 1982). In order to reach the conclusion that superior performance on small numerosity tasks is an indication of a greater understanding of small numerosities than of large numerosities, it is necessary to include conditions that permit us to distinguish quantifier solutions from operator solutions.

\section{REFERENCES}

Blevins, B., Mace, P., Cooper, R., Starkey, P., \& Leitner, E. (1981, April). What do children know about addition and subtraction? Paper presented at the meeting of the Society for Research in Child Development, Boston.

Bryant, P. (1974). Perception and understanding in young children. New York: Basic Books.
CHI, M. T. H., \& KLAR, D. (1975). Span and rate of apprehension in children and adults. Journal of Experimental Child Psychology, 19, 434-439.

COOPER, R. (1984). Early number development: Discovering number space with addition and subtraction. In C. Sophian (Ed.), Origins of cognitive skill (pp. 157-192). Hillsdale, NJ: Erlbaum.

Cooper, R. G., CAmpbell, R., \& Blevins, B. (1983). Numerical representation from infancy to middle childhood: What develops? In D. R. Rogers \& J. A. Sloboda (Eds.), The acquisition of symbolic skills. New York: Plenum Press.

COOPER, R. G., STARKEY, P. (1977, August). What preschoolers know about number: Does subitizing develop? Paper presented at the meeting of the American Psychological Association, San Francisco.

Cooper, R. G., Starkey, P., Blevins, B., Goth, P., \& Leitner, E. (1978, June). Number development: Addition and subtraction. Paper presented at the Jean Piaget Society meetings, Philadelphia.

Flavell, J. (1970). Concept development. In P. Mussen (Ed.), Carmichael's manual of child psychology (pp. 983-1059). New York: Wiley.

GelmaN, R. (1972). The nature and development of early number concepts. In H. W. Reese \& L. P. Lipsitt (Eds.), Advances in child development and behavior (Vol. 7, pp. 115-167). New York: Academic Press.

GelmaN, R. (1982). Accessing one-to-one correspondence: Still another paper on conservation. British Journal of Psychology, 73, 209-220.

Gelman, R., \& Baillargeon, R. (1983). A review of some Piagetian concepts. In J. Flavell \& E. Markman (Eds.), Handbook of child psychology: Vol. 3. Cognitive development (pp. 167-230). New York: Wiley.

Gelman, R., \& Gallistel, C. R. (1978). The child's understanding of number. Cambridge, MA: Harvard University Press.

PiAGET, J. (1952). The child's conception of number. New York: Norton. SIEGLER, R. (1986). Children's thinking. Englewood Cliffs, NJ: Prentice-Hall.

SiEgLER, R. S., \& Robinson, M. (1982). The development of numerical understanding. In H. W. Reese \& L. P. Lipsitt (Eds.), Advances in child development and behavior (Vol. 16, pp. 241-312). New York: Academic Press.

Winer, G. A. (1974). Conservation of different quantities among preschool children. Child Development, 45, 839-842. 